\section{LA VACCINAZIONE ANTI-COVID... NELLO SPAZIO E NEL TEMPO}

$$
c=v_{W} *\left(\frac{10}{3}+a \ln \left(10-W_{f}\right)^{n}\right)+f_{1}\left(W_{f}-9\right)^{5}+f_{2}\left(W_{f}-9\right)^{11}
$$

Gli esperti di Star Trek avranno riconosciuto in questa espressione la formula complessa del warp speed, la velocità di curvatura diventata il simbolo della corsa al vaccino Covid (vedi Donald Trump). Questa formula descrive la curvatura dello spazio/tempo che permette di viaggiare oltre la velocità della luce. Almeno così dicono.

Lo ignorava Federico Marchetti, diversamente da Donald Trump, quando, nel lontano maggio 2020, esponeva ai lettori di Medico e Bambino lo stato di avanzamento della ricerca sui vaccini. Deformando spazio e tempo, le aziende biotecnologiche, in poche settimane, hanno identificato il virus, pubblicato il suo genoma, individuato la proteina target per un vaccino, sviluppato una serie di test per la diagnosi (PCR, antigenico su saliva o tampone nasale, esalato nasale....) e per la sierodiagnosi e poi, ma questo è il più facile, sviluppato il vaccino e dimostrato che può funzionare. Anzi, russi e cinesi, meno problematizzanti di noi occidentali, già vaccinano. Almeno così dicono.

Adepto del pensiero lento (vedi Kahneman e Tversky) indugiavo sulla storia della reazione di Wasserman o sullo sviluppo e sulla diffusione del test alla tubercolina e dei vaccini mai arrivati per sifilide, tbc o malaria, pensando ai tempi che Covid avrebbe richiesto.

Invece, abbiamo vinto!

Forse, no, forse la partita non è ancora finita. Forse la distorsione dello spazio/tempo è stata eccessiva, forse ora molte cose le sappiamo, ma molte ancora no.

\section{Tanti corrono, quanti arriveranno?}

La corsa è iniziata a febbraio-marzo e i partecipanti sono stati subito molti, oggi forse più di 200. Ma la corsa a un vaccino virale richiede particolari procedure, fra cui quelle di colture cellulari per la riproduzione del virus, come si fa ancora per l'influenza, che certamente molte delle biotech che lavorano sul Covid non hanno. In realtà molta parte dello sviluppo è stata fatta su base bioinformatica, il che ha permesso di bruciare le prime tappe. Molte piccole aziende, che di per sé non avrebbero i mezzi di sviluppare un vaccino, possono avere buone idee per creare nuove piattaforme e sperare di venderle a Big pharma. Molte aziende sono saltate sul carro di Covid mentre stavano sviluppando nuove piattaforme per altri vaccini. Vediamo sul sito di Moderna la loro pipeline, vi sono diversi prodotti in sviluppo su cui puntavano. Covid ha offerto l'occasione di accedere a ingenti fondi pubblici per validare la loro piattaforma. Penso che potrebbero anche vendere il vaccino a un prezzo politico, visto i soldi che già hanno ricevuto, ma avranno validato una piattaforma che potrebbe rivoluzionare il modo di fare vaccini. Altre aziende possono avere una prospettiva locale e pensare di coprire il fabbisogno nazionale senza dover fare contratti con Big pharma. Il panorama sarà complesso e imporrà delle scelte.
Ma meglio avere l'imbarazzo della scelta che non avere scelte (ne parleremo dopo).

Due aziende hanno completato la fase 3 reclutando un grande numero di volontari sani trattati con vaccino o placebo e in pochi mesi hanno ora due dati importanti. II vaccino è sicuro a breve termine, il vaccino è efficace a breve termine. Questo è il risultato di analisi ad interim, nessuno dei protocolli che potete vedere sul sito clinicaltrials.gov prevede di terminare prima di uno o due anni dopo la somministrazione dell'ultima dose. L'osservazione procede per valutare effetti a più lungo termine.

Almeno altri due vaccini sono in fase avanzata Johnson\&Johnson, Astra Zeneca) con piattaforme diverse. II panorama si complicherà nei prossimi mesi. Per non parlare di vaccini fantasma come Sputnik o i vari cinesi che pare siano già autorizzati per l'uso e forse già usati. Per ora sembra che le Autorità regolatorie in occidente abbiano resistito alle pressioni politiche per approvazioni affrettate, Pfizer ha aspettato le elezioni. Personalmente, non confido che lo stesso avvenga in Russia o in Cina che chiaramente puntano al vaccino come strumento di penetrazione politica. La Cina annuncia di avere già dei contratti con vari Paesi asiatici e forse anche con il Brasile.

\section{Qualcuno arriverà, ma noi da dove partiamo?}

Girano notizie che ritengo insensate, tanto da non credere alla loro affidabilità. L'Italia ha comprato 40 milioni di dosi del vaccino Astra Zeneca (qualcuno ha confermato?), I'Europa ha comprato 300 milioni di dosi del vaccino Pfizer (2). La curvatura dello spazio/tempo mi sembra eccessiva, potremmo scivolare e trovarci in un buco nero.

I dati diffusi da Moderna e Pfizer sono notevoli, ma valgono per i comunicati stampa. Scientificamente, vorremmo saperne di più. Prima di tutto, vorremmo che fossero consolidati con osservazioni più prolungate, vorremmo avere elaborazioni più precise, stratificate e per obiettivi primari e secondari. Vorremmo sapere se il vaccino riduce la mortalità, la progressione dell'infezione verso forme gravi, la frequenza di casi gravi o lievi o se riesce anche a prevenire il contagio. I dati devono essere sottoposti alle Autorità regolatorie in maniera molto dettagliata. Questo non può avvenire in pochi giorni.

Ci sono alcuni motivi per cui, secondo me, non possiamo pensare a una vaccinazione di massa nel 2021 . Il primo è che le aziende non hanno la capacità produttiva necessaria, il secondo è che con dati di efficacia/sicurezza a breve termine non è sensato vaccinare tutti. Il terzo è che le Aziende non hanno intenzione di chiedere questo tipo di autorizzazione. Infatti, per Pfizer e Moderna si parla di EUA: Emergency Use Authorization. Questo significa che il vaccino potrà essere usato in condizioni circoscritte, sulla base delle informazioni disponibili a novembre 2020 e in attesa di ulteriori dati. Si pongono questioni non secondarie di responsabilità. Le Aziende non possono fornire dati di sicurezza né di efficacia a lungo termine perché non li hanno. Se i Governi fanno pressione per avere, comunque, il vaccino devono assumersi un carico di responsabilità.

Si può fare? L'analisi dei costi/benefici è diversa se si pen- 
sa a una vaccinazione mirata o a una vaccinazione universale. Quindi, possiamo pensare a vaccinazioni di gruppi a rischio, come personale sanitario o necessario in emergenze, oppure a una vaccinazione "ad anello" cioè dei contatti di un malato. In questi casi, i rischi prevenuti possono compensare la mancanza di informazioni. Potremmo anche accontentarci di un'immunità di pochi mesi o anche rischiare eventi avversi non emersi nelle sperimentazioni cliniche.

Vorremmo avere dati sugli anziani (almeno io vorrei saperne qualcosa!), in genere più difficili da immunizzare, quale vaccino offrirà una minore protezione. Dato molto rilevante perché la letalità, molto bassa nel giovane, raggiunge il $25-30 \%$ sopra gli 80 anni.

Visto che i vaccini disponibili sono almeno due dobbiamo decidere come iniziare una strategia mirata. Non abbiamo dati sulla loro interscambiabilità, potremo fare dei richiami usandoli alternativamente? La scelta iniziale rischia di vincolarci per i prossimi anni.

\section{Il vaccino e le strategie di sanità pubblica}

In attesa di ulteriori dati, il vaccino sarà uno degli strumenti di una strategia di Sanità pubblica più complessa. Le norme in atto e l'intervento sul territorio non potranno essere abbandonati fino a quando non riusciremo a ottenere con il vaccino e con la diffusione del virus non voluta, ma inevitabile, un'immunità di popolazione.

Per passare a una vaccinazione allargata, o universale, dobbiamo verificare l'efficacia sul campo e la capacità del sistema, oggi sostanzialmente tarato sulle vaccinazioni universali dell'infanzia, di somministrare 40-80 milioni di dosi. In Italia, negli ultimi anni, oltre ai vaccini per l'infanzia abbiamo solo esperienza per influenza e per HPV, non certo brillanti. Per l'epatite $B$ ci eravamo dati un obiettivo più a lungo termine vaccinando nuovi nati e dodicenni oltre che gruppi a rischio. Se pensiamo di abbattere la circolazione del Covid con una massiccia campagna di vaccina- zione dobbiamo prepararla bene. Il che significa anche far funzionare l'anagrafe vaccinale nazionale, rafforzare la capacità di individuare focolai e circoscriverli, fare dei controlli sieroepidemiologici, creare un sistema di sorveglianza dedicato con l'obbligo di denuncia e rete di sorveglianza magari analoga a quella per le epatiti (SEIEVA) o per l'influenza (Influnet).

\section{I compiti del vaccino}

Al vaccino possiamo chiedere di controllare l'epidemia, di essere uno strumento di routine per un'infezione che diventa endemica con riaccensioni epidemiche, di eliminare la malattia, di eradicare il virus. Un vaccino in grado di impedire l'infezione permetterebbe di interrompere la catena di trasmissione sostenuta da un grande pool di asintomatici. Un vaccino che riduce la mortalità, ma non il contagio, non permette di raggiungere questo obiettivo.

Per interrompere la circolazione del virus bisogna raggiungere coperture elevate. Dovremo, infine, pensare se introdurre l'obbligo di vaccinazione almeno per certe categorie. Non si entra in un college americano senza essere vaccinati almeno per meningococco, ma spesso chiedono molto altro. Renderemo obbligatorio il vaccino per i liceali, per gli universitari?

Vista la diffusibilità del virus questo non potrà essere solo un obiettivo nazionale. Come stiamo facendo per la poliomielite dovremo garantire un livello di immunità di popolazione che possa impedire un eventuale ritorno dell'infezione da altri Paesi a bassa copertura vaccinale. Oppure, come per la poliomielite, vaccinare tutto il mondo. Ma qui la formula curva/spazio diventa ancora più complessa.

Baroukh M. Assael

Responsabile scientifico della rivista Il giornale della vaccinazione. Ed Minerva Medica Consulente AIFA per i farmaci per la fibrosi cistica già Direttore del Centro Fibrosi Cistica di Verona 